INRA Prod. Anim.,

2008, $21(1), 127-136$

\section{Les vecteurs viraux : outils modernes de vaccination}

S. BERTAGNOLI' , B. PIGNOLETI', S. BIACCHESI ${ }^{2}$, M. ELOIT ${ }^{3}$, B. KLONJKOWSKI ${ }^{3}$,

J. RICHARDSON ${ }^{3}$, M. BREMONT ${ }^{2}$

${ }^{1}$ INRA, Université de Toulouse, ENVT, UMR1225 Interactions Hôtes-Agents Pathogènes, F-31076 Toulouse, France

2 INRA,UR892 Virologie et Immunologie Moléculaires, F-78352 Jouy-en-Josas, France

3 INRA, AFSSA, ENVA, UMR1161 Virologie, F-94704 Maisons-Alfort, France

Courriel : s.bertagnoli@envt.fr

La vaccination est un acte médical, individuel ou intégré dans une stratégie de prophylaxie collective, s'appuyant sur des outils, les vaccins, évalués pour leur efficacité et leur innocuité. Dans certains cas, des aspects éthiques et réglementaires imposent des contraintes supplémentaires orientant les choix technologiques. Pour faire face aux défis actuels de la médecine vétérinaire, l'utilisation de vecteurs viraux est une alternative prometteuse.

La prévention des maladies animales d'intérêt économique fait appel en France à deux types de stratégie non exclusives et souvent complémentaires : la prophylaxie sanitaire et la prophylaxie médicale. Cette dernière s'appuie sur l'emploi de vaccins sûrs et efficaces dont la mise au point nécessite de nombreux travaux de recherchedéveloppement. C'est dans ce cadre que l'INRA, et en particulier le Département de Santé Animale, a depuis de nombreuses années orienté les efforts de recherche vers l'élaboration de vaccins de nouvelle génération tenant compte des exigences réglementaires et sociétales et se fondant sur les dernières avancées scientifiques du domaine. En effet, la vaccinologie est une science moderne en perpétuelle évolution alimentée par les progrès constants réalisés en immunologie, infectiologie et biotechnologies.

Ainsi, depuis les premiers pas de la vaccination (contre la variole, puis la rage) de très nombreux vaccins ont été mis au point, et ceci suivant deux grands principes : préparation à partir de produits biologiques vivants, ou bien de produits biologiques inactivés. Classiquement, on distingue alors deux types de vaccins : les vaccins à agents vivants atténués, et les vaccins à agents inactivés (ou tués). Une troisième catégorie de vaccins dits «classiques» a par la suite été développée : les vaccins «sous-unité». Dans ce cas, les principes actifs sont constitués par des composants qui représentent des fractions immunologiquement actives des microorganismes. Ces vaccins, au même titre que ceux à agents inactivés, entrent dans la catégorie des vaccins à agents inertes. Actuellement, les techniques issues de l'ingénierie génétique tendent à considérablement simplifier la préparation des vaccins de type sous-unité. En effet, des antigènes dits recombinants peuvent facilement être produits par des systèmes d'expression procaryote ou eucaryote. Il faut au préalable identifier le ou les antigènes susceptibles d'induire une réponse immune protectrice, et intégrer leur gène dans le génome de la cellule ou du virus (bactérie, levure, cellule de mammifère, baculovirus...) qui assurera leur production in vitro. La purification de la protéine d'intérêt sera alors bien plus facile et plus sûre que par le passé.

Les techniques de génie génétique ont actuellement un impact majeur sur la préparation des vaccins vétérinaires. Elles autorisent la mise au point de vaccins de nouvelles générations, non seulement de type sous-unité, mais aussi de type vivant atténué. Ainsi voit-on maintenant arriver sur le marché des vaccins vectorisés recombinants : le gène codant pour la protéine d'intérêt est inséré au sein du génome d'un virus ou d'une bactérie apathogènes. Ce vecteur vivant, dénommé vecteur vaccinal, exprimera alors l'an- tigène étranger dans l'organisme hôte au cours de l'infection vaccinale, induisant ainsi une réponse immunitaire que l'on souhaite protectrice. De nombreux micro-organismes ont été ainsi génétiquement modifiés à des fins vaccinales : salmonelles, E.coli, et surtout divers virus. Classiquement, ceux-ci appartenaient à des familles virales à ADN (surtout poxvirus, et adenovirus), mais les progrès réalisés dans la maîtrise des outils de biologie moléculaire (génétique inverse en particulier) ont permis d'étendre la liste des vecteurs vaccinaux à certains virus à ARN (dont les rhabdovirus et les paramyxovirus). Nous présenterons dans cette revue des exemples remarquables d'études, par des équipes INRA, de vecteurs viraux destinés à vacciner diverses espèces animales contre des agents pathogènes majeurs. Mais si ces vaccins recombinés sont très attractifs en raison de leur facilité de production, leur efficacité, leur faible coût de revient, ou leur facilité d'emploi, il n'en demeure pas moins que certains problèmes relatifs à leur pouvoir pathogène résiduel, ou aux possibilités de recombinaison avec des souches sauvages sont évoqués. C'est pourquoi nous verrons également que la recherche s'oriente actuellement vers la mise au point de vecteurs viraux non réplicatifs (donc dépourvus de risque infectieux pour l'animal) pour une espèce cible (ou un ensemble d'espèces). 


\section{1 / Le virus myxomateux, nouveau poxvirus vecteur vaccinal}

Les recherches développées depuis de nombreuses années sur l'étude de la myxomatose et du virus myxomateux (poxvirus spécifique du lapin européen) au sein de l'Unité Mixte de Recherche INRA-ENVT Interactions Hôtes-Agents Pathogènes ayant débouché, à la fin des années 70 , sur la mise au point de la souche vaccinale SG33 actuellement commercialisée en France (Saurat et al 1978), c'est tout naturellement que la thématique des vecteurs vaccinaux utilisant des poxvirus animaux a été initiée au début des années 90. En effet, parmi les nombreux virus susceptibles de jouer le rôle de vecteur vaccinal, le système le mieux connu et le plus courant reste celui fondé sur l'utilisation de poxvirus infectieux recombinés. De nombreux gènes issus de micro-organismes divers (virus de l'hépatite $\mathrm{B}$, de la rage, de la fièvre aphteuse, de la grippe, du sida...) ont été introduits dans le génome du virus de la vaccine, poxvirus modèle et emblématique, à des fins vaccinales. D'autres poxvirus, dont des souches modifiées de la vaccine, des poxvirus aviaires ou de ruminants, et bien sûr le virus myxomateux pour l'INRA et l'ENVT (Messud-Petit et Bertagnoli 2000), ont aussi été utilisés plus tardivement en tant que vecteurs vaccinaux, avec en général le même succès (Drillien et al 2003). Le principe d'obtention des poxvirus recombinés est commun à tous les genres utilisés jusqu'ici et s'appuie sur des caractéristiques particulières du cycle des poxvirus, leur conférant de nombreux avantages mais aussi quelques limites (Drillien et al 2003).

\section{1 / Méthodologie, avantages et limites des poxvirus en tant que vecteurs viraux}

\section{a) Schéma général}

Les poxvirus ont un génome ADN de grande taille et non infectieux par luimême, ce qui impose de construire le vecteur viral exprimant un antigène vaccinal en deux étapes : construction d'un plasmide vecteur d'insertion contenant le gène (transgène) du candidat vaccin, puis transfert du transgène au virus par recombinaison homologue au sein d'une cellule transfectée et infectée (figure 1, Cox et al 1992).

Figure 1. Principe d'obtention d'un poxvirus recombiné.

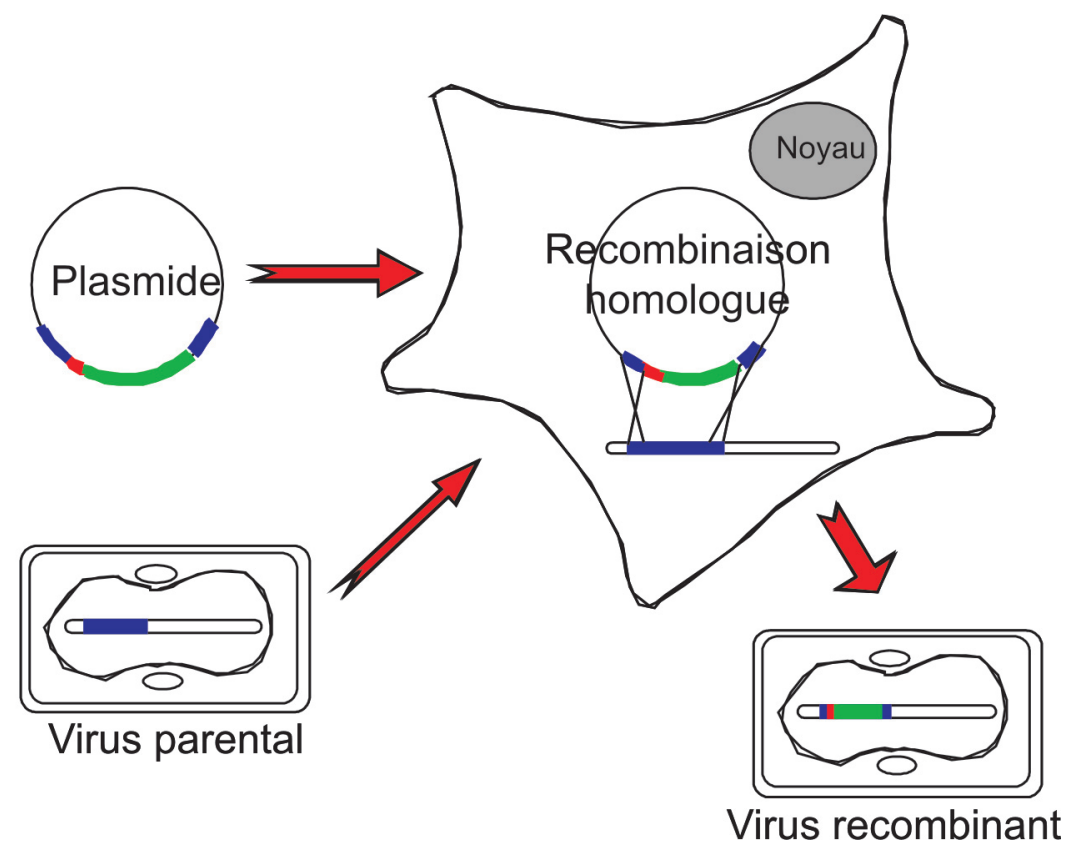

Les séquences flanquantes dirigeant la recombinaison sont figurées en bleu, le transgène (séquence codant pour l'antigène vaccinal) en vert et le promoteur poxviral (indispensable à l'expression du gène) en rouge. La recombinaison homologue a lieu dans le cytoplasme de la cellule, le virus recombinant produit contenant dans son génome le gène d'intérêt.

\section{b) Avantages}

La réplication des poxvirus étant strictement cytoplasmique, les complications éventuelles d'intégration dans le génome de la cellule hôte sont fortement improbables. Le marquage, la détection et la purification des protéines exprimées en sont également relativement aisés. Le génome viral, de grande taille, tolère facilement l'insertion de grandes quantités d'ADN étranger (plus de $25 \mathrm{~kb}$ ) (Smith et Moss 1983). L'antigène vaccinal produit par la cellule infectée par le poxvirus vecteur est très proche de la protéine native, et son activité est en général parfaitement conservée.

Bien que quelques complications post-vaccinales aient été décrites, le virus de la vaccine reste très sûr et efficace puisqu'il a permis l'éradication mondiale de la variole. Certains variants du virus de la vaccine présentent en plus des caractéristiques qui augmentent son innocuité (Sutter et Moss 1992). On peut également aujourd'hui utiliser d'autres poxvirus totalement apathogènes pour l'homme (en particulier les poxvirus aviaires) (Drillien et al 2003).

Le coût de production de la plupart des poxvirus est faible : cela est surtout vrai pour le virus de la vaccine qui peut se multiplier sur de très nombreux supports (diverses cellules, nombreuses espèces animales). De plus, les poxvirus recombinés étant des vaccins vivants, la dose protectrice minimale est réduite. Les poxvirus sont en général très stables : ils supportent bien la congélation, la lyophilisation et la réhydratation sans perte importante du titre infectieux. Cela les rend particulièrement adaptés aux vaccinations de masse. Enfin, les poxvirus sont faciles à administrer, puisque la voie intradermique est la méthode classique d'inoculation (par scarification, injection ou système à air comprimé). Il est également possible, dans certains cas, d'utiliser la voie orale (Blancou et al 1986).

\section{c) Limites}

Tout d'abord, les poxvirus détruisent à terme les cellules qu'ils infectent. Ils ne peuvent donc être utilisés que pour une expression transitoire de la protéine étrangère. Mais c'est surtout le problème du pouvoir pathogène résiduel qui peut limiter leur emploi en tant que vecteurs vaccinaux, en particulier pour des populations immunodéprimées (sida de l'Homme par exemple) pour lesquelles le risque de complications post-vaccinales est accru. La solution réside en la maîtrise des facteurs de pathogénicité et l'exploitation de nouvelles souches totalement apathogènes du virus de la vaccine, ou d'autres poxvirus à spectre d'hôte étroit (poxvirus aviaire par exemple) (Drillien et al 2003). Parmi ceux-ci, certains offrent 
l'avantage de pouvoir vacciner contre au moins deux maladies. Par exemple, des virus recombinés fondés sur l'utilisation d'un poxvirus de ruminant atténué peuvent protéger le bétail contre la dermatose nodulaire contagieuse bovine (une poxvirose tropicale) et un autre agent infectieux ciblé (Berhe et al 2003). L'emploi de tels vaccins est généralement envisagé pour bénéficier des avantages des vaccins vivants, quand il est impossible d'atténuer le pouvoir pathogène de l'agent ciblé, quand des quantités suffisantes de micro-organismes ne peuvent pas être produites aisément, ou encore, lorsque l'on veut simultanément vacciner contre plusieurs agents pathogènes.

\section{2 / Application à la vaccination du lapin européen}

Les considérations générales précédentes correspondent parfaitement aux préoccupations de la filière cunicole depuis le début des années 90. En effet, deux maladies virales constituent un danger majeur pour les populations de lapins européens : la myxomatose et la maladie hémorragique virale du lapin (RVHD). En plus d'un déséquilibre écologique préjudiciable lorsqu'elles touchent les lapins de garenne, elles sont responsables de pertes économiques non négligeables en élevage. Pour lutter contre ces maladies, divers moyens peuvent être employés, parmi lesquels la vaccination occupe une place de choix. Actuellement certains vaccins antimyxomateux (vaccins à virus atténués) et anti-RVHD (vaccin à virus inactivés) existent sur le marché, chacun tentant de répondre aux exigences des producteurs de lapins. Bien qu'efficaces, ils présentent néanmoins quelques inconvénients rendant leur utilisation coûteuse ou peu aisée. La souche vaccinale atténuée de virus myxomateux SG33 possède un pouvoir immunodépresseur résiduel pouvant être préjudiciable en élevage fermier à faible niveau d'hygiène. Le vaccin antiRVHD est produit à partir de broyats d'organes de lapins infectés, seul moyen actuel de multiplier le virus ; sa production est donc lourde et coûteuse. Pourtant, l'antigène majeur du RHDV (protéine VP60), est nécessaire et suffisante pour assurer une protection efficace (Laurent et al 1994, Bertagnoli et al 1996b). Afin d'améliorer l'efficacité des vaccins existant contre la RVHD, un programme de recherche visant à exprimer la protéine VP60 dans des systèmes recombinants vivants de type poxvirus a été initié au début des années 90 sous l'impulsion du Département de Santé Animale de l'INRA, en partenariat avec l'ONCFS et I'ITAVI'. En utilisant un virus recombiné myxomatose-RHDV, il était légitime d'espérer vacciner simultanément les lapins contre la myxomatose et la RVHD. Il semblait possible, en choisissant des sites de recombinaison appropriés, d'améliorer l'innocuité du virus SG33 pour les jeunes lapins, en inactivant des gènes codant des facteurs de pathogénicité précédemment identifiés. Afin de protéger le réservoir naturel de ces deux viroses - les lapins de garenne - il était important de pouvoir démontrer l'efficacité de l'administration du vecteur vaccinal par voie orale. Une seule injection intradermique de ces virus a permis de protéger les jeunes lapins contre une épreuve virulente par un virus sauvage de la RVHD, dans les conditions utilisées pour les contrôles vaccinaux en France, et ce de façon aussi efficace que les préparations vaccinales inactivées du commerce (Bertagnoli et al 1996a). L'utilisation d'un virus vecteur recombiné par voie orale, bien que nécessitant une plus forte dose, a également induit une protection parfaite du jeune lapin (Messud-Petit et Bertagnoli 2000). Cette protection s'établit rapidement, dès 5 jours après l'injection, ou 15 jours après administration orale du virus. S'agissant de la vaccination contre la myxomatose, une seule injection par voie intradermique de virus recombiné donne le même niveau de protection contre la myxomatose que le virus vaccinal SG33. Il en est de même d'une administration unique de forte dose par voie orale. Cette protection est certes partielle, certains lapins succombant à l'épreuve virulente et d'autres exprimant une forme atténuée de la maladie, mais certainement suffisante pour fournir une couverture vaccinale efficace au niveau des élevages. L'immunogénicité de la souche myxomateuse vaccinale SG33 ne semble pas avoir été modifiée par l'insertion d'un gène étranger. Aucun signe clinique de myxomatose, signe d'un pouvoir pathogène résiduel pour les lapereaux, n'a été observé après l'inoculation des virus recombinés. Néanmoins, ces résultats doivent être confirmés dans des conditions d'élevage soumis à une plus grande pression microbienne.
Ces travaux nous indiquent donc que le virus myxomateux représente un vecteur poxviral potentiel efficace dont l'application vaccinale mérite d'être étendue aux différents agents infectieux préjudiciables à la filière cunicole.

Enfin, étant donné les résultats obtenus avec les poxvirus aviaires qui sont d'excellents vecteurs viraux non réplicatifs pour les mammifères, un développement du virus myxomateux en tant que vecteur vaccinal chez d'autres mammifères que le lapin pourrait être envisageable.

\section{3 / Perspectives : application à la vaccination d'autres espèces d'intérêt}

Les stratégies vaccinales fondées sur l'utilisation de poxvirus recombinés ont beaucoup évolué ces dernières années, en raison principalement d'un souci de sécurité d'emploi des vecteurs viraux. En effet, la possibilité d'accidents vaccinaux liés au pouvoir pathogène résiduel des souches «historiques» du virus de la vaccine a stimulé la recherche de vecteurs poxviraux alternatifs plus sûrs d'emploi. Rapidement deux pistes ont été explorées avec succès : 1'élaboration de souches du virus de la vaccine ayant perdu leurs capacités réplicatives chez les mammifères (dont l'Homme) (NYVAC, MVA) et l'utilisation de poxvirus aviaires qui par définition sont incapables de se multiplier chez les mammifères (Drillien et al 2003). Le concept de vecteur poxviral non réplicatif est alors apparu et cette stratégie s'est largement répandue. Actuellement, des vaccins recombinés issus de canaripoxvirus sont commercialisés en médecine vétérinaire (vaccination contre la grippe équine ou la leucose féline). C'est dans ce cadre que de nouveaux candidats vecteurs peuvent être recherchés afin d'améliorer les réponses immunitaires obtenues, en particulier en permettant d'élaborer de nouveaux protocoles vaccinaux (alternance de vecteurs viraux selon la technique du «prime-boost $\left.\left.{ }^{2}\right\rangle\right)$ ou de bénéficier des avantages potentiels et spécifiques de chaque poxvirus. La possibilité d'employer le virus myxomateux comme vecteur vaccinal non réplicatif chez les ruminants a ainsi été évaluée dans un programme de recherche débuté en 2003 et soutenu par le Département de Santé Animale de I'INRA et l'AFSSA (Agence française

1 ONCFS : Office National de la Chasse et de la Faune Sauvage ; ITAVI : Institut Technique de l'Aviculture.

2 «Prime-boost» : technique d'immunisation alternant des vecteurs vaccinaux différents exprimant le même antigène, selon des protocoles variables (voie et nombre d'administrations) 
de Sécurité Sanitaire des Aliments). Il a été clairement mis en évidence que le virus myxomateux pouvait infecter des lignées cellulaires, ainsi que des fibroblastes primaires et des lymphocytes, d'origine bovine et ovine et exprimer des transgènes tout en accomplissant un cycle abortif (Pignolet et al 2007). Des essais in vivo d'infection de moutons par voie intradermique ont confirmé l'innocuité du virus myxomateux, mais ont aussi révélé sa capacité à induire une réponse inflammatoire au point d'inoculation et surtout une réponse immunitaire spécifique contre l'antigène exprimé par le virus recombiné.

Ces résultats, bien que préliminaires, sont encourageants pour le développement de vaccins non réplicatifs fondés sur l'utilisation du virus myxomateux chez les ruminants. Cependant l'efficacité de cette stratégie vaccinale ne pourra vraiment s'apprécier que par des tests de protection de bovins ou d'ovins face à une épreuve virulente pour une maladie d'intérêt.

\section{2 / Adenovirus humains et animaux défectifs : une nouvelle génération de vec- teurs viraux}

Les adénovirus (Ad) sont de petits virus nus à $\mathrm{ADN}$, classiquement utilisés en tant que vecteurs vaccinaux. L'Unité Mixte de Recherche INRAAFSSA-ENVA de Virologie travaille activement depuis de nombreuses années à l'amélioration de vecteurs adénoviraux et a ainsi initié des activités de recherche visant à les modifier de manière à les rendre «défectifs», c'est à dire incapables de se multiplier in vivo. Il s'agit conceptuellement d'une «vaccination génétique» où le virus vecteur délivre le gène exogène aux cellules cibles sans effectuer de cycle productif et sans lyse cellulaire. L'antigène vaccinal est alors exprimé et fait l'objet d'une présentation adéquate au système immunitaire en association avec des molécules du complexe majeur d'histocompatibilité (CMH).

\section{1 / Application à la vaccination animale : utilisation des adéno- virus humains}

Dans une première phase du travail, les avantages/inconvénients relatifs à la biosécurité (Oualikene et al 1994) et l'efficacité (Ganne et al 1994) des adé- novirus humains, aussi bien dans des modèles murins que dans des espèces cibles potentielles (carnivores, porcins, bovins, chèvres) ont été documentés (Adam et al 1994, Eloit et Adam 1995, Gonin et al 1996). La vaccination des nouveau-nés, y compris en présence d'anticorps d'origine maternelle, s'est révélée remarquablement efficace (Le Pottier et al 1997, Monteil et al 1997).

Des développements spécifiques ont eu lieu en matière de vaccins vis-à-vis de différents agents pathogènes :

- Le virus du l'immunodéficience féline $(F I V)$ représente un excellent modèle du virus du sida humain (HIV). Le programme initié au sein de l'UMR de Virologie dans les années 90 a visé à construire puis à tester différents virus recombinants fondés sur l'Ad humain exprimant des antigènes du virus FIV (Gonin et al 1995). Bien qu'ils soient capables d'induire une réponse anticorps détectable, leur efficacité en matière de protection s'est révélée très médiocre.

- La péritonite infectieuse féline à coronavirus félin, outre sa grande importance en pathologie féline, est une maladie de physiopathologie très complexe proche de celle de la dengue chez l'homme. Tous les essais de vaccination par voie parentérale, utilisant des vaccins à virus vivants ou inactivés, se sont soldés par des échecs, les animaux vaccinés mourant habituellement plus rapidement que les animaux non vaccinés. Des Ad recombinants exprimant le gène de la glycoprotéine membranaire $\mathrm{M}$ ont été construits et testés. Les résultats obtenus sont suffisamment encourageants, un premier essai ayant permis de protéger $50 \%$ des chats contre une épreuve virulente tuant $100 \%$ des témoins (Gonin et al 1996), pour envisager de poursuivre des études avec de nouvelles constructions virales (adénovirus canin (Cav), meilleure définition des immunogènes) (Gonon et al 1999).

Compte tenu du potentiel désormais démontré des vecteurs adénoviraux pour une vaccination par voie locale (nasale), un programme collaboratif avec l'Université de Liège a été initié. Les gènes $\mathrm{gD}$ et $\mathrm{gC}$ de l'herpesvirus bovin de la rhinotrachéite infectieuse bovine ont été insérés dans le génome d'Ad sous le contrôle de différents promoteurs, ce qui a permis l'induction d'un bon niveau d'immunité muqueuse et de protection après administration par voie nasale chez les bovins (Gogev et al 2002). Des essais ont également été conduits dans un modèle expéri- mental de Leptospirose chez la gerbille (collaboration avec le laboratoire de G. André-Fontaine à Nantes) (Branger et al 2001).

\section{2 / Application à la vaccination par voie orale}

L'UMR Virologie s'est rapidement intéressée au développement de vaccins administrés par voie orale, en raison de leur facilité d'administration et de leur potentiel à induire des Réponses Immunitaires (RI) à la fois dans les compartiments systémique et muqueux. Les vecteurs vaccinaux dérivés des Ad se sont avérés capables d'induire d'excellentes RI à médiation humorale et cellulaire après administration parentérale. Néanmoins, l'administration orale des types d'Ad les plus étudiés, tels que ceux dérivés du sérotype 5 , semble moins efficace, en particulier lorsqu'ils sont non réplicatifs. Lors de l'administration orale de vaccins à base d'Ad, il est probable que l'induction de la RI est limitée par, d'une part, le franchissement de l'épithélium digestif et, d'autre part, la prise en charge d'antigène par les cellules présentatrices d'antigènes (CPA) professionnelles. Ces limites relèvent du tropisme des $\mathrm{Ad}$, qui est déterminé en grande partie par une des protéines de la capside virale, la fibre, en raison de ses interactions de haute affinité avec certains récepteurs cellulaires. En théorie, le tropisme intestinal des Ad pourrait faire l'objet d'une optimisation importante par la modification ou le remplacement de la fibre. Une cinquantaine de sérotypes d'adénovirus humains, répartis dans six sous-groupes (A-F), ont été identifiés à ce jour. Ils sont responsables de diverses pathologies spécifiques de tissu, en relation avec l'utilisation de différents récepteurs cellulaires. Il est plausible que cette diversité puisse être exploitée afin d'optimiser les Ad comme vaccins administrés par voie orale. Il semblait ainsi pertinent de caractériser les interactions entre des Ad ayant différents tropismes et la muqueuse digestive et d'évaluer leurs conséquences pour l'induction des RI après vaccination par voie orale. L'influence du tropisme du vecteur vaccinal sur l'amplitude et la qualité des RI induites présente un intérêt à la fois fondamental (mécanismes de l'induction des RI muqueuses) et finalisé (nouvelles stratégies de vaccination par voie muqueuse).

Les études ont été menées à l'aide d'une banque de vecteurs dérivés d'Ad5. Ces vecteurs sont identiques, hormis pour la fibre qui est remplacée 
par la fibre naturelle de différents sérotypes humains. Tous les vecteurs portent le même transgène, codant un antigène modèle (la luciférase). Les expériences d'infections de lignées épithéliales intestinales ont mis en évidence d'importantes différences entre les sous-groupes d'Ad quant à leur capacité à $\mathrm{s}$ 'attacher à et à transduire ${ }^{3}$ des épithéliums intestinaux différenciés. En particulier, par rapport à un vecteur qui porte la fibre de sérotype 5, certains Ad munis de fibres des sous-groupes B et $\mathrm{D}$ ont présenté un tropisme accru pour la muqueuse digestive humaine. Ces résultats suggèrent que le transfert de gènes à l'intestin humain pourrait faire l'objet d'importantes améliorations en exploitant le tropisme de sérotypes existants d'Ad humains (Lecollinet et al 2006).

Des expériences de vaccination de souris par voie intragastrique ont révélé que tous les vecteurs testés sont capables d'induire des réponses humorales locales contre l'antigène modèle utilisé. Néanmoins, des différences dans la cinétique et l'amplitude des réponses immunes induites par les différents vecteurs ont pu être mises en évidence, même pour des vecteurs partageant les mêmes récepteurs. L'analyse de l'expression du transgène dans différents tissus n'a décelé, de manière inattendue, aucune relation entre capacité à transduire la muqueuse intestinale et à induire une réponse humorale locale. Ces études in vivo suggèrent que l'interaction entre la fibre et son récepteur spécifique sur des cellules épithéliales n'est pas une étape limitante pour l'induction des RI après administration par voie orale, ce qui peut signifier que ce sont les étapes ultérieures au franchissement qui seraient déterminantes.

\section{3 / Vaccination animale et adé- novirus xénogéniques}

Depuis une dizaine d'année, l'UMR Virologie conduit un programme d'étude de certains Ad animaux (porcin et canin) dont l'objectif est de maîtriser la génétique de ces virus et la manipulation de leur génome, et d'évaluer leur aptitude à servir de vecteurs de transfert de gène pour le développement de vaccins recombinants utilisables en médecine vétérinaire.

Des nombreux essais vaccinaux conduits utilisant des vecteurs réplica- tifs ou non, il ressort que les vecteurs réplicatifs sont quantitativement (diminution de la dose efficace) et qualitativement (induction d'une immunité muqueuse) particulièrement efficaces. Néanmoins, ces vecteurs présentent l'inconvénient majeur d'être associés, chez l'hôte permissif, à une production de particules infectieuses pouvant être excrétées dans l'environnement.

Le premier vecteur ainsi construit dérive du sérotype 3 de l'adénovirus porcin (Pav3) et exprime une glycoprotéine $(\mathrm{gD})$ du virus de la maladie d'Aujeszky (ou pseudorage). L'efficacité de ce vecteur réplicatif porcin a pu être démontrée au cours d'un essai de vaccination par voie orale de porcs conventionnels. Lors de cet essai, réalisé en milieu confiné, la diffusion du vecteur réplicatif a été évaluée en laissant des animaux sentinelles au contact des animaux immunisés. L'absence de séroconversion des animaux sentinelles suggère que le vecteur réplicatif porcin est incapable d'être transmis d'un animal à l'autre. Ces résultats encourageants permettent d'envisager d'étendre l'utilisation de ces vecteurs porcins à la vaccination d'espèces sauvages (sangliers), en particulier pour la lutte contre la Peste Porcine Classique, une infection dont le sanglier est un réservoir pour le porc domestique. Le vecteur vaccinal exprimant un antigène de ce virus (gp55) étant d'ores et déjà disponible sous les formes réplicative et non réplicative, l'efficacité relative des deux vecteurs pourra être estimée, ainsi que l'efficacité du vecteur vaccinal en fonction du protocole d'immunisation (une administration comparée à deux). Néanmoins, un point limitant actuel de ce vecteur reste la difficulté de le produire en grande quantité pour une exploitation industrielle.

Nous avons plus récemment prolongé ces efforts en développant des vecteurs dérivés de la souche vaccinale Manhattan de l'adénovirus canin type 2 (Cav2). Des vecteurs réplicatif et non réplicatif ont été obtenus à partir de Cav2 exprimant la glycoprotéine du virus de la rage et utilisés dans des essais d'immunisation de nombreuses espèces telles que la souris, le chien, le chat, le singe et le mouton. Les résultats obtenus montrent l'efficacité d'une unique administration pour induire une réponse humorale par voie parentérale (toutes espèces) ou orale (souris et chiens). De plus, l'administration parentérale s'accompagne d'une réponse immunitaire à médiation cellulaire (souris et singe).

\section{4 / Perspectives : adenovirus animaux semi-réplicatifs}

Les vecteurs adénoviraux réplicatifs présentent un risque potentiel de diffusion entre individus et leur usage éventuel reste donc limité, dans une optique générale de limitation de la diffusion d'Organismes Génétiquement Modifiés (OGM). Les vecteurs non réplicatifs, par contre, sont, en principe, sûrs au plan du risque biologique et certains d'entre eux se révèlent efficaces par voie orale ; néanmoins, les doses utilisées pour obtenir une protection sont souvent peu compatibles avec leur utilisation comme vaccin. L'UMR INRA-AFSSA-ENVA Virologie envisage ainsi de poursuivre le développement de vecteurs semi-réplicatifs dérivés du sérotype 2 de l'adénovirus canin (Cav2) afin de diminuer les risques de diffusion dans l'environnement et de transmission à l'Homme. Ces vecteurs resteront capables de répliquer leur génome sans générer de particules virales infectieuses soit dans le cadre du maintien de la barrière d'espèces soit par délétions génomiques ciblées.

\section{3 / Les rhabdovirus de pois- sons, vecteurs viraux aty- piques}

L'unité INRA de Virologie et Immunologie Moléculaires de Jouy-enJosas s'est depuis de nombreuses années spécialisée dans l'étude de virus responsables de maladies des poissons et plus particulièrement des rhabdovirus (virus enveloppés, à ARN simple brin non segmenté de polarité négative). Parmi eux, les virus de la nécrose hématopoḯtique infectieuse (vNHI) et de la septicémie hémorragique virale (vSHV) sont des agents pathogènes majeurs responsables de pertes économiques importantes pour les élevages piscicoles. Ces deux virus, totalement différents sur le plan antigénique, infectent les truites et les saumons de tout âge et induisent une maladie aboutissant à de nombreuses lésions hémorragiques et à la mort de 70 à $100 \%$ des animaux infectés en l'espace de 8 à 20 jours.

\footnotetext{
3 Transduction : transfert de gène dans une cellule par un vecteur viral capable ou non de se répliquer de façon autonome, mais sans production de néo-particules virales ; la cellule transduite exprimera ainsi les séquences portées par le vecteur.
} 


\section{1 / Avantages et méthodologie}

\section{a) Avantages}

Les rhabdovirus offrent plusieurs avantages en tant que vecteurs transitoires d'expression d'antigènes vaccinaux : $i)$ leur réplication est exclusivement cytoplasmique sans aucun risque d'intégration de leur information génétique dans le génome de la cellule hôte, ii) leur génome est stable ce qui réduit les risques de recombinaison et de perte des séquences non essentielles, iii) ils permettent l'insertion d'un ou plusieurs gènes additionnels et l'échange d'un ou plusieurs de leurs gènes afin de modifier leur tropisme, iv) l'expression du gène d'intérêt est modulable en fonction de la méthode d'insertion choisie, v) l'insertion d'un ou plusieurs gènes s'accompagne d'une atténuation du pouvoir pathogène viral.

b) Méthodologie : le système de génétique inverse pour le virus NHI

L'ARN génomique des rhabdovirus n'est jamais nu dans les cellules infectées, il est toujours associé à des protéines essentielles (nucléoprotéine, $\mathrm{N}$; phosphoprotéine, $\mathrm{P}$ et polymérase, L) pour former le complexe réplicatif permettant la transcription et la réplication du génome. On ne sait pas manipuler directement l'ARN, il faut passer par des intermédiaires ADN complémentaires (ADNc) qui par transcription redonnent de l'ARN. C'est ce que l'on appelle la génétique inverse. L'équipe de Conzelmann en 1994 (Schnell et al 1994) a élaboré pour la première fois pour le virus de la rage un tel système basé sur l'utilisation de quatre constructions plasmidiques et d'un virus recombinant de la vaccine (vTF7-3) exprimant une enzyme, l'ARN polymérase $\mathrm{T} 7$ (figure $2 \mathrm{~A}$ ).

Une approche similaire a été développée pour le virus NHI. Ainsi, quatre constructions plasmidiques sont nécessaires (figure 2A). Un plasmide pIHN, contenant une copie ADNc du génome du vNHI (environ 12 Kbases) qui par transcription fournit un ARN copie conforme du génome viral. Trois autres plasmides (pT7-N, pT7-P et pT7-L) contenant respectivement les gènes codant pour N, P et L permettent l'expression des protéines correspondantes. Ces 4 constructions plasmidiques sont sous contrôle d'un promoteur T7. Lorsque des cellules préalablement infectées par vTF7-3 (qui fournit l'ARN polymérase T7) sont transfectées avec ces quatre plasmides, il y a synthèse de l'ARN viral (issu du pIHN)
Figure 2. A. Système de génétique inverse : explications dans le texte (Abréviations : prom $T 7$ : séquence promotrice de l'ARN polymérase $T 7$; Ribo : séquence ribozyme permettant de cliver l'ARN génomique produit dans la cellule après action de l'ARN polymérase $T 7$; tT7 : séquence d'arrêt de l'ARN polymérase T7 ; vTF7-3 : virus recombinant de la vaccine exprimant l'ARN polymérase T7).

B. Truitelles infectées par bain avec le VNHI-LUC (génome schématisé avec localisation du gène LUC en région intergénique) observées à 8 heures post-infection. Les points colorés signalent l'expression de la luciférase et ainsi localisent le virus d'épreuve : on remarque ainsi que la base des nageoires constitue la porte d'entrée primaire du virus

A

pNHI

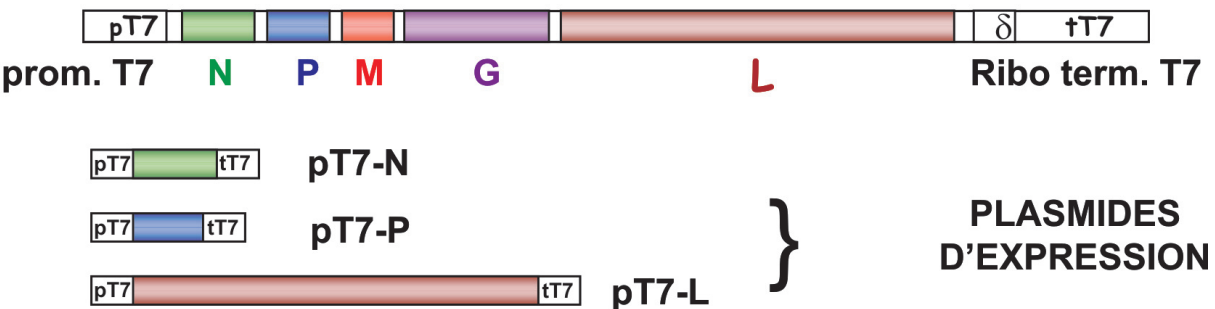

2 TRANSFECTION

1 INFECTION vTF7-3

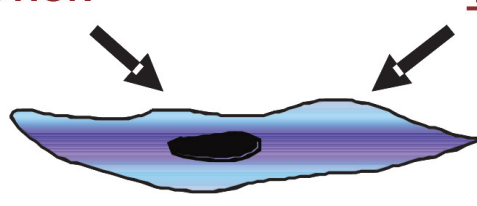

CELLULES

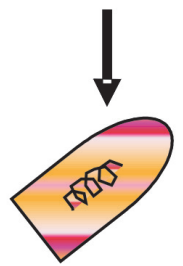

VIRUS RECOMBINANT

B

vNHI-LUC

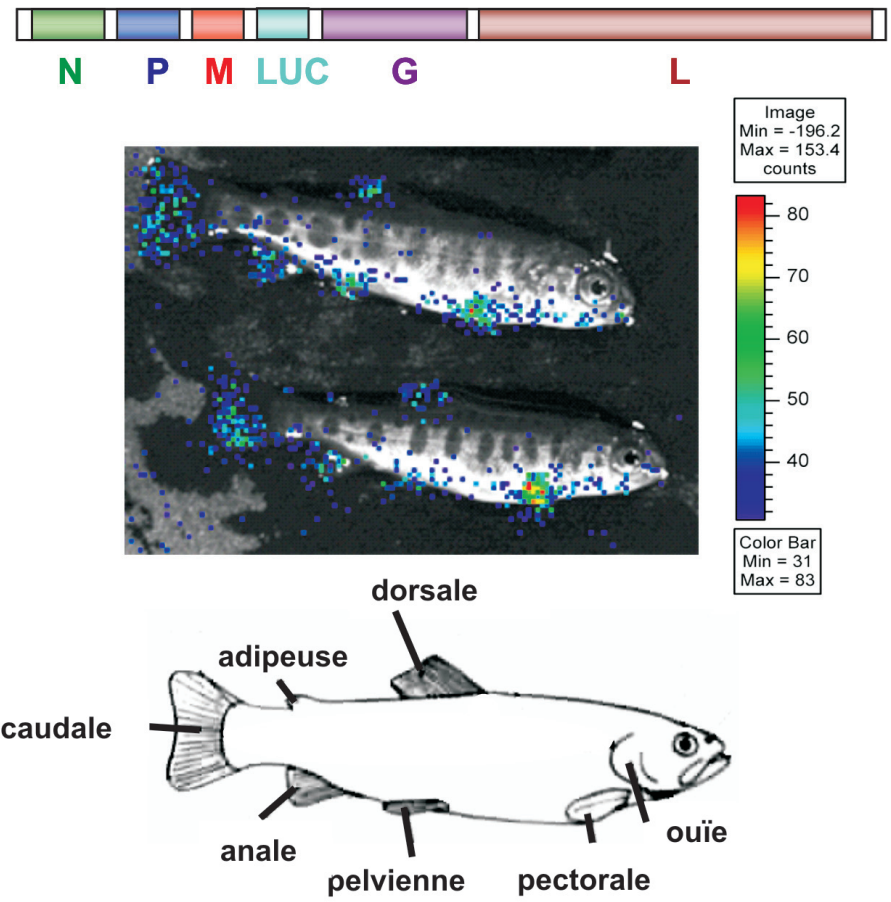


qui est encapsidé par les 3 protéines du complexe réplicatif $\mathrm{N}, \mathrm{P}$ et $\mathrm{L}$. On reconstitue donc artificiellement un complexe réplicatif et le cycle de réplication ainsi déclenché se poursuit jusqu'à la formation de particules virales qui bourgeonnent à la membrane cellulaire et sont relarguées dans le milieu de culture (Biacchesi et al 2000a, 2000b, Brémont 2005).

\section{2 / Le vNHI comme vecteur de gènes}

a) Application à la vaccination contre les rhabdovirus des poissons: échange de la glycoprotéine de surface

Nous avons étudié la possibilité d'échanger la glycoprotéine $\mathrm{G}$ du vNHI par celle d'un autre rhabdovirus antigéniquement distinct : le vSHV. Bien que le pourcentage d'identité entre ces deux glycoprotéines soit inférieur à $40 \%$, elles présentent plusieurs caractéristiques communes : i) la $\mathrm{G}$ est la seule protéine responsable de la synthèse d'anticorps neutralisants chez les animaux infectés, ii) des mutations ciblées sur la glycoprotéine permettent d'atténuer la virulence, iii) la $\mathrm{G}$ est responsable de l'attachement du virus à son récepteur cellulaire. Un virus recombinant (vNHI-Gvshv) dans lequel le gène $\mathrm{G} \mathrm{du} v$ NHI était remplacé par celui du vSHV, a été obtenu, et l'incorporation de la glycoprotéine étrangère dans les particules virales recombinantes a pu être démontrée. Le vecteur recombinant se réplique aussi bien que les virus d'origine ( $v$ NHI et vSHV) en culture de cellules et est également pathogène pour les poissons. Ce résultat montre qu'il est possible d'incorporer une glycoprotéine étrangère dans le vNHI, ce qui est un préalable indispensable à la génération à terme d'un vaccin bivalent (exprimant 2 glycoprotéines) permettant ainsi de lutter simultanément contre les deux virus vSHV et vNHI (cf. plus bas) (Biacchesi et al 2002).

Parallèlement à ce travail, il a été montré que la délétion dans le génome viral d'un petit gène NV, codant pour une protéine non structurale, permettait la production de virus vNHI- $\Delta \mathrm{NV}$ totalement apathogène (Thoulouze et al 2004).

L'ensemble de ces résultats nous a conduit à produire des virus vNHI recombinant dont le génome est délété du gène $\mathrm{NV}$ et remplacé par le gène codant la $\mathrm{G}$ du vSHV. Nous obtenons donc des virus recombinants chimériques $\mathrm{vNHI}-\Delta \mathrm{NV}-\mathrm{G}_{\mathrm{SHV}}$, totalement apathogènes et qui expriment simultané- ment à la surface des virions les 2 glycoprotéines externes du vNHI et du vSHV. Ces virus chimériques sont donc potentiellement utilisables comme souche vaccinale vivante bivalente pour protéger simultanément contre le vSHV et le vNHI, ces deux agents pathogènes importants qui cohabitent dans les élevages piscicoles français (Brevet : Brémont M., Biacchesi S., Leberre M., Thoulouze M.I., Utilisation de Novirhabdovirus modifiés pour l'obtention de vaccins, $\left.\mathrm{n}^{\circ} 0205702,2002\right)$.

b) Application à la vaccination multivalente par ajout d'un gène additionnel : mise au point et perspectives

Afin d'évaluer la possibilité de construire des vecteurs vNHI exprimant un ou plusieurs gènes surnuméraires, le génome de vNHI a été modifié par l'insertion dans une région intergénique, $\mathrm{du}$ gène rapporteur luciférase (LUC) qui permet des mesures d'expression extrêmement sensibles. Un virus recombinant vNHI-LUC a été produit en culture cellulaire, et, malgré l'augmentation de la taille du nouveau génome obtenu, il a des caractéristiques de multiplication en culture cellulaire (cinétique de réplication, effet cytopathogène, titre viral...) semblables à celles du virus sauvage (Harmache et al 2006). Ce virus est aussi pathogène chez la truite que le virus sauvage, ce qui atteste la viabilité de la construction obtenue in vivo, et in fine constitue la meilleure preuve de faisabilité de la technique. La méthode de détection extrêmement sensible de l'expression du gène de la luciférase a permis de développer un système d'imagerie par bioluminescence permettant de visualiser in vivo la réplication du virus chez les truites infectées par balnéation : une caméra extrêmement sensible capable de détecter les photons émis par la luciférase est couplée à un système informatique de reconstitution d'image. Nous avons ainsi pu établir une cinétique d'infection, visualiser les étapes très précoces de la réplication virale chez l'animal infecté et enfin, déterminer la porte d'entrée du virus (figure $2 \mathrm{~B}$ ). La réplication du vNHI, reflétée par l'expression de la luciférase, est visualisée chez la truite à différents temps après l'infection. A 8 et $16 \mathrm{~h}$, la réplication virale est localisée à la base des nageoires qui constituent donc la porte primaire d'entrée du virus.

De nombreux virus recombinants dérivés du vNHI, dans le génome duquel un à trois gènes surnuméraires ont été introduits, ont ainsi été produits. Par exemple, des truitelles immunisées par balnéation avec un vNHI recombinant modifié par insertions intergéniques exprimant les protéines d'enveloppe $\mathrm{HE}$ et $\mathrm{F}$ du virus de l'anémie infectieuse du saumon, (ISAV,) ne présentent aucun signe clinique lié à cette primo-infection et sont totalement protégés d'une infection par les virus sauvage ISAV et IHNV. Ainsi certains constituent d'ores et déjà de très bons outils vaccinaux chez les salmonidés, n'attendant qu'un développement commercial, puisque l'insertion simultanée de plusieurs cassettes d'expression a pour effet d'atténuer de façon satisfaisante le vNHI.

Par ailleurs, ces vNHI recombinants constituent également de bons outils vaccinaux pour les vertébrés supérieurs. En injectant chez la souris le vNHI-LUC, incapable de se multiplier à des températures supérieures à $20^{\circ} \mathrm{C}$, nous avons fait la démonstration qu'il est naturellement inactivé et qu'il joue un rôle d'adjuvant naturel. Des expériences sont en cours pour évaluer la réponse immune chez la souris immunisée par un vNHI exprimant les deux antigènes HA et NA du virus de la grippe (Brevet : Harmache A, Brémont M., Développement de Novirhabdovirus comme vecteur de gènes, $\mathrm{n}^{\circ}$ 06290982.5, 2006).

\section{Conclusion}

L'INRA, par l'intermédiaire des unités propres ou des UMR du Département de Santé Animale, participe activement à l'amélioration des outils vaccinaux disponibles, en particulier par la mise au point de nouveaux vecteurs viraux. Nous venons de voir que le champ d'investigation est important et que les résultats obtenus, bien que destinés initialement à la vaccination d'espèces animales d'intérêt économique, sont potentiellement valorisables dans le cadre de la pathologie comparée (cas des chats et de la PIF ou du FIV). Un trait commun aux recherches menées par les différentes équipes est le souci d'accroître la sécurité d'emploi des vecteurs viraux étudiés en tendant, lorsque cela était possible, vers l'élaboration de vecteurs non réplicatifs ou semi-réplicatifs. Ceux-ci pourront trouver une application vaccinale pour des espèces animales de choix pour lesquelles un des objectifs affichés par l'INRA est de mettre au point des plates-formes technologiques éprouvées, s'appuyant sur des vecteurs préalablement validés. Celles-ci pourront être mobilisables rapidement en cas d'émergence de maladies considérées comme majeures et contre lesquelles la vaccination est requise. 


\section{Références}

Adam M., Le Pottier M.F., Eloit M., 1994. Vaccination of pigs with replication defective adenovirus vectored vaccine: the example of pseudorabies. Vet. Microbiol., 42, 205-215.

Berhe G., Minet C., Le Goff C., Barrett T., Ngangnou A., Grillet C., Libeau G., Fleming M., Black D.N., Diallo A., 2003. Development of a dual recombinant vaccine to protect small ruminants against peste-des-petits-ruminants virus and capripoxvirus infections. J. Virol., 77, 1571-1577.

Bertagnoli S., Gelfi J., Le Gall G., Boilletot E., Vautherot J. F., Rasschaert D., Laurent S., Petit F., Boucraut-Baralon C., Milon A., 1996a. Protection against myxomatosis and rabbit viral hemorrhagic disease with recombinant myxoma viruses expressing rabbit hemorrhagic disease virus capsid protein. J. Virol., 70, 5061-5066.

Bertagnoli S., Gelfi J., Petit F., Vautherot J.F., Rasschaert D., Laurent S., Le Gall G., Boilletot E., Chantal J., Boucraut-Baralon C., 1996b. Protection of rabbits against Rabbit Viral Hemorrhagic disease with a vaccinia-RHDV recombinant virus. Vaccine, 14, 506-510.

Biacchesi S., Yu Y.X., Bearzotti M., Tafalla C., Fernandez-Alonso M., Brémont M., 2000a. Rescue of synthetic salmonid rhabdovirus minigenomes. J. Gen. Virol., 81, 1941-1945.

Biacchesi S., Thoulouze M.I., Bearzotti M., Yu Y.X., Brémont M., 2000b. Recovery of NV knockout infectious hematopoietic necrosis virus expressing foreign genes. J. Virol., 74, 1124711253.

Biacchesi S., Bearzotti M., Bouguyon E., Brémont M., 2002. Heterologous exchanges of the glycoprotein and the matrix protein in a Novirhabdovirus. J. Virol., 76, 2881-2889.

Blancou J., Kieny M.P., Lathe R., Lecocq J.P., Pastoret P.P., Soulebot J.P., Desmettre P., 1986. Oral vaccination of the fox against rabies using a live recombinant vaccinia virus. Nature, 322, 373-375.

Branger C., Sonrier C., Chatrenet B., Klonjkowski B., Ruvoen-Clouet N., Aubert A., Andre-Fontaine G., Eloit M., 2001. Identification of the hemolysis-associated protein 1 as a crossprotective immunogen of Leptospira interrogans by adenovirus-mediated vaccination. Infect. Immun., 69, 6831-6838.

Brémont M., 2005. Reverse genetics on fish rhabdoviruses: tools to study the pathogenesis of fish rhabdoviruses. Curr. Top. Microbiol. Immunol., 292, 119-141.
Cox W.I., Tartaglia J., Paoletti E., 1992. Poxviruses recombinant as live vaccines. In: Recombinant poxviruses. Binns M.M., Smith G.L. (Ed), CRC Press, Boca Raton, USA, 123162.

Drillien R., Spehner, D., Autran B., Garin D., 2003. Les poxvirus : une famille de vecteurs. Virologie, 7, 243-253.

Eloit M., Adam M., 1995. Isogenic adenoviruses type 5 expressing or not expressing the E1A gene: efficiency as virus vectors in the vaccination of permissive and non permissive species. J. Gen. Virol., 76, 1583-1589.

Ganne V., Eloit M., Laval A., Adam M., Trouve G., 1994. Enhancement of the efficacy of a replication defective adenovirus vectored vaccine by the addition of oil adjuvants. Vaccine, 12, 1190-1196.

Gogev S., Vanderheijden N., Lemaire M. Schynts F., D'offay J., Deprez I., Adam M., Eloit M., Thiry E., 2002. Induction of protective immunity to bovine herpesvirus type 1 in cattle by intranasal administration of replication-defective human adenovirus type 5 expressing glycoprotein $\mathrm{gC}$ or $\mathrm{gD}$. Vaccine, 20, 1451-1465.

Gonin P., Fournier A., Oualikene W. Moraillon A., Eloit M., 1995. Immunization trial of cats with a replication-defective adenovirus type 5 expressing the Env gene of feline immunodeficiency virus. Vet. Microbiol., 45, 394-401.

Gonin P., Oualikene O., Fournier A., Soulier M., Audonnet J.C., Riviere M., Eloit M., 1996. Evaluation of a replication-defective adenovirus expressing the feline infectious peritonitis membrane protein as a vaccine in cats. Vaccine Res., 4, 217-227.

Gonon V., Duquesne V., Klonjkowski B., Monteil M., Aubert A., Eloit M., 1999. Clearance of infection in cats naturally infected with feline coronaviruses is associated with antiglycoprotein S antibody response, J. Gen. Virol., 80, 2315-2317.

Harmache A., LeBerre M., Droineau S., Giovannini M., Brémont M., 2006 Bioluminescence imaging on live infected salmonids reveals that the fin bases are the major portal of entry for Novirhabdovirus. J. Virol., 80, 3655-3659.

Laurent S., Vautherot J.F., Madelaine M.F., Le Gall G., Rasschaert D., 1994. Recombinant rabbit hemorrhagic disease virus capsid protein expressed in baculovirus self-assembles into viruslike particles and induces protection. J. Virol., 68, 6794-6798.

Lecollinet S., Gavard F., Havenga M.J.E., Spiller O.B., Lemckert A., Goudsmit J., Eloit M., Richardson J., 2006. Improved gene delivery to intestinal mucosa by adenoviral vectors bearing subgroup B and D fibers. J. Virol., 80, 27472759.

Le Pottier M.F., Monteil M., Houdayer C., Eloit M., 1997. Study of the delivery of the $\mathrm{gD}$ gene of PRV to one-day-old piglets by adenovirus or plasmid DNA as ways to by-pass the inhibition of immune response by colostral antibodies. Vet. Microbiol., 55, 75-80.

Messud-Petit F., Bertagnoli S., 2000 . Le virus myxomateux : de l'agent pathogène au vecteur vaccinal. Virologie, 4, 453-462.

Oualikene W., Gonin P., Eloit M., 1994. Short and long term dissemination of wild type and deletion mutants of adenovirus type 5 in mouse and cotton rat. J. Gen. Virol., 75, 2765-2768.

Monteil M., Le Potier M.F., Cariolet R., Houdayer C., Eloit M., 1997. Effective priming of neonates born to immune dams against the pseudorabies virus glycoprotein $\mathrm{gD}$ by replication-incompetent adenovirus-mediated gene transfer at birth. J. Gen. Virol., 78, 3303-3310.

Pignolet B., Duteyrat J.L., Allemandou A., Gelfi J., Foucras G., Bertagnoli S., 2007. In vitro permissivity of bovine cells for wild-type and vaccinal Myxoma virus strains. Virol. J., 4, 9499.

Saurat P., Gilbert Y., Ganiere J.P., 1978. Etude d'une souche de virus myxomateux modifié. Revue Méd. Vét., 129, 415-451.

Schnell M.J., Mebastsion T., Conzelmann K.K., 1994. Infectious rabies viruses from cloned cDNA. EMBO J., 13, 4195-4203.

Smith G.L., Moss B., 1983. Infectious poxvirus vectors have capacity for at least 25000 base pairs of foreign DNA. Gene, 25, 21-28.

Sutter G., Moss B., 1992. Nonreplicating vaccinia vector efficiently expresses recombinant genes. Proc. Natl. Acad. Sci. USA, 89, 1084710851

Thoulouze M.I., Bouguyon E., Carpentier C., Brémont M., 2004. Essential role of the NV protein of Novirhabdovirus for pathogenicity in rainbow trout. J. Virol. 78, 4098-4107.

\section{Résumé}

Les vaccins destinés aux animaux appartiennent à deux grandes catégories : les vaccins à agents vivants, et ceux à agents inertes. Depuis quelques années, dans chacune de ces catégories, les innovations technologiques ont considérablement amélioré et diversifié les stratégies vaccinales disponibles en fonction des contraintes liées à des préoccupations tant d'innocuité, que d'efficacité ou encore de nature économique. C'est dans ce cadre que l'INRA a depuis de nombreuses années orienté les efforts de recherche vers l'élaboration de nouveaux vaccins s'appuyant sur la mise au point de vecteurs viraux adaptés à diverses espèces animales et susceptibles de répondre aux exigences des filières animales. Dans cette revue, nous décrivons ainsi les principes d'obtention et le développement de vecteurs vaccinaux fondés sur l'emploi de poxvirus animaux à spectre d'hôte étroit (virus myxomateux), d'adenovirus humains ou animaux défectifs (c'est-à-dire ayant perdu toute capacité à se multiplier chez l'hôte) ainsi que de rhabdovirus de poissons modifiés par génétique inverse. Des exemples d'application de vaccination non seulement contre des maladies animales d'intérêt économique, mais aussi dans le cadre de modèles de pathologie comparée permettent d'illustrer le potentiel indiscutable de ces vecteurs viraux et d'envisager leur emploi pour le contrôle de maladies animales émergentes ou réémergentes en Europe. 


\begin{abstract}
Viral vectors: new tools for vaccination

Vaccines designed for animal use belong to two main categories: vaccines with alive agents and those with inactive agents. Over the last few years, in each category, technological innovations have considerably improved and diversified vaccination strategies in order to meet new demands for better protection, efficacy and economic performances. In this context, INRA has directed its research efforts towards the development of vaccines based on the elaboration of viral vectors adapted to a variety of animal species and likely to meet the demand of animal industries. This review presents a description of how vaccine vectors using animal poxviruses with a narrow host spectrum (myxoma virus), human or animal defective adenoviruses (meaning they can no longer multiply within the host) as well as fish rhabdoviruses modified by reverse genetics, can be obtained and developed. Examples of possible vaccination applications, not only against economically important animal diseases but also as part of compared pathology models, illustrate the unquestionable potential of these viral vectors and allow considering their use in control programmes of emerging or reemerging animal diseases in Europe.
\end{abstract}

BERTAGNOLI S., PIGNOLET B., BIACCHESI S., ELOIT M., KLONJKOWSKI B,, RICHARDSON J., BREMONT M., 2008. Les vecteurs viraux : outils modernes de vaccination. INRA Prod. Anim., 21, 127-136. 
